\title{
Context-Aware Systems and Applications
}

\author{
Emil Vassev • Son Vuong
}

Published online: 12 March 2014

(C) Springer Science+Business Media New York 2014

\section{Foreword}

This special issue, with five papers extended from selected contributions to The 1st International Conference on Context-Aware Systems and Applications (ICCASA 2012) held in Ho Chi Minh City of Vietnam, will serve as a reference material for researchers, scientists, professionals and students in computer science and computer engineering as well as developers and practitioners in computing and networking systems design by providing them with state-of-the-art research findings and future opportunities and trends. These papers include some recent advances in context-awareness reflected in this special issue. In particular, the special issue covers various aspects of context-awareness as follows:

Paper 1 by Kaiyu Wan and Vangalur Alagar provides the current state of the art of Cyber-Physical Systems (CPS) security, identifies the issues surrounding secure control, and investigates the extent to which context information may be used to improve security and survivability of CPS.

Paper 2 by Phan Cong Vinh presents agent-based ubiquitous computing (AUC), a form of distributed computing, by which computational processes are executed concurrently by assigning each computational process to one of agents on a ubiquitous computing system (UCS). The overarching goal of AUC is to support the seamless integration of information and services. To this end, taking advantage of the categorical structures, this

E. Vassev $(\bowtie)$

Lero at University of Limerick, Limerick, Ireland

e-mail: emil@vassev.com

\section{S. Vuong}

University of British Columbia, Vancouver, Canada

e-mail: svuong@cs.ubc.ca

paper develops a firm formal approach to formalize the AUC for information and service integration.

Paper 3 by Angelo Furno and Eugenio Zimeo proposes a design approach based on a semantic model for context representation. It is an extension of the OWL-S ontology aimed at enriching the expressiveness of each section of a typical OWL-S semantic service description, by means of context conditions and adaptation rules. By having access to continuously updated context information, these descriptions can be exploited by a discovery/composition tool to automatically find the atomic or composite services that can be bettertuned to the requestor's behaviors and to the particular situations of the surrounding environment.

Paper 4 by Nguyen Thanh Tung proposes two methods to optimize the lifetime of chain-based protocols using Integer Linear Programming (ILP) formulations. Also, a method to determine the bounds of the lifetime for any energy-efficient routing protocol is presented. Furthermore, previous researches assume that the base station position is randomly placed without optimization. In this paper, a non-convex optimization model is developed for solving the base station location optimization problem.

Paper 5 by Areerat Songwattana, Thanaruk Theeramunkong and Phan Cong Vinh presents a socalled Learning Based Replacement (LBR) algorithm, a hybrid approach towards an efficient replacement model for web caching by incorporating a machine learning technique (naive Bayes) into the Least Recently Used (LRU) replacement method to improve prediction of possibility that an existing page will be revised by a succeeding request, from access history in a web log.

We owe our deepest gratitude to Dr. Nguyen Manh Hung-Chairman and Rector of Nguyen Tat Thanh University in Vietnam for his useful support, especially to all the authors for their valuable contribution to this special issue and their great efforts, and also to the 
referees for ensuring the high quality of the material presented here. All of them are extremely professional and cooperative. We wish to express our thanks to the Editor-in-Chief, Prof. Imrich Chlamtac, for his important assistance with the process of assembling the special issue.

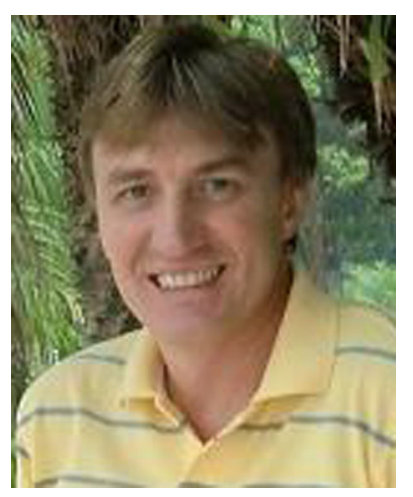

Dr. Emil Vassev received his M.Sc. in Computer Science (2005) and his Ph.D. in Computer Science (2008) from Concordia University, Montreal, Canada. Currently, he is a research fellow at Lero (the Irish Software Engineering Research Centre) at University of Limerick, Ireland where he is leading the Lero's participation in the ASCENS FP7 project and the Lero's joint project with ESA on Autonomous Software Systems Development Approaches. His research focuses on knowledge representation and awareness for self-adaptive systems. A part from the main research, Dr. Vassev's research interests include engineering autonomic systems, distributed computing, formal methods, cyber-physical systems and software engineering. He has published two books and over 100 internationally peer-reviewed papers. As part of his collaboration with NASA, Vassev has been awarded one patent with another one pending.

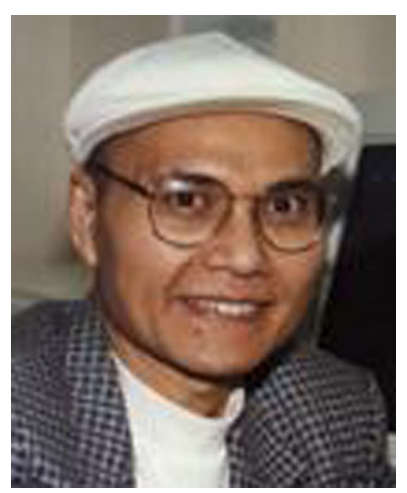

Prof. Dr. Son Vuong received his B.S. in Electrical Engineering from California State University, Sacramento, USA; M.Eng. in Systems Engineering at Carleton University in Ottawa, Canada; and Ph.D. in Computer Science from the University of Waterloo, Canada where he was a lecturer and assistant professor in 19811982. Since 1983, he has been a professor of Computer Science at the University of British Columbia in Vancouver, Canada, where he was a founder of the Distributed System Research Group and is now Director of the Laboratory for Networks and Internet Computing (NICLab). He is an international renowned researcher on protocol engineering, distributed multimedia systems, and collaborative computing. His areas of research interest in recent years include the Internet of Things, ambient networks, grid and $\mathrm{P} 2 \mathrm{P}$ computing, $\mathrm{P} 2 \mathrm{P}$ video streaming, network security and mobile computing and mobile learning.

He has (co) authored a US patent, 200 papers and co-edited three books, including the book on "Recent Advances in Distributed Multimedia Systems" published in 1999. He has supervised thesis research of 80 graduate $(\mathrm{PhD}$ and $\mathrm{MSc}$ ) students. He was a co-leader of the major $\$ 30 \mathrm{M}$ grant proposal to establish a new Network of Centres of Excellence in 1999, called Global Information Systems and Software Technology_ GISST that made to the final short list. He served on many conference program committees and was general or technical (co)chair and (co)organizer of ten international conferences (NCAS'11, ACM Multimedia'08, DMS'08, IEEE NOMS'06, DMS'97, ICDCS'95, PSTV'94, FORTE'89, IWPTS'88). He served on the Canadian National Science and Engineering Research Council (NSERC) Grant Selection Committee in 1999-2003, and also serves on the Board of Directors of two high-tech companies. 